\title{
Proliferation and differentiation of mouse embryonic stem cells lacking all lamins
}

Cell Research (2013) 23:1420-1423. doi:10.1038/cr.2013.118; published online 27 August 2013

\section{Dear Editor,}

Lamins are the major components of the nuclear lamina (NL), which is located underneath the inner nuclear membrane [1]. Diverse array of functions have been assigned to lamins, and alterations of lamins are associated with a number of human diseases [2]. Of the three lamin genes in mammals, Lmna expresses all isoforms of Atype lamins, referred to as lamin-A/C, through alternative splicing, while Lmnb1 and Lmnb2 express B-type lamins. Despite subtype-specific sequence variations and different expression patterns, lamin proteins exhibit overall sequence similarity. Therefore, a potential functional redundancy among different lamin proteins has made it difficult to precisely address the role of lamins in mammals.

Early studies indicate that A-type lamins are not expressed in ESCs or pre-implantation stage embryos [3, 4]. Recently, although one study showed no detectable lamin-A/C expression in either wild-type or lamin-B1 and -B2 double knockout (lamin-B DKO) mouse ESCs [5], another reported the expression of low levels of lamin$\mathrm{A} / \mathrm{C}$ in several mouse ESC lines and in pre-implantation embryos [6]. The proper self-renewal and differentiation of lamin-B DKO ESCs in vitro suggest that lamins are not required for basic cell survival at least in ESCs [5]. The low level of lamin-A/C expression, however, has called into the question whether lamins are indeed dispensable for the survival of ESCs. The potential redundancy among lamin proteins in mammals makes it necessary to evaluate lamins' functions in cells or animals deleted of all three lamin genes. Here, we report that ESCs lacking all lamins undergo proper self-renewal and differentiation as judged by marker expression. Our findings are consistent with a role of lamins in tissue building and maintenance. The creation of lamin triple knockout ESCs also offers the field an important tool to further study the role of these important nuclear proteins in genome organization during development and tissue homeostasis.

Since lamin-A/C appear to be expressed in a number of mouse ESCs and in pre-implantation embryos as detected by more sensitive methods [6], we analyzed lamin- $\mathrm{A} / \mathrm{C}$ expression based on the genome-wide mRNAsequencing (RNA-seq) datasets from wild-type ESCs and MEFs (Kim et al., unpublished). After normalizing by total sequence reads, sequence tags corresponding to Lmna were plotted (Supplementary information, Figure S1A). We found that wild-type ESCs indeed express a very low level of Lmna compared to MEFs. This low level of expression may explain why Lmna transcripts or proteins in ESCs are not easily detected in previous studies.

The expression of lamin-A/C in ESCs raises an important question regarding whether lamin- $\mathrm{A} / \mathrm{C}$ compensate for the function of lamin-B in lamin-B DKO $\left(L_{m n b 1^{-/}}\right.$ ;Lmnb2 $2^{--}$) ESCs. To address this question, we mutated both copies of Lmna alleles in $L m n b 1^{-\alpha} ; L m n b 2^{-/}$ESCs by two rounds of gene targeting. To remove all isoforms of lamin-A/C, we designed two gene-targeting constructs containing different selection markers to delete a region from exon 2 to the first 14 bases of exon 6, which encodes a region spanning the $\alpha$-helical rod domain of lamin-A/C (Figure 1A). In the first round of targeting, the deletion was introduced into $L m n b 1^{-/} ; \mathrm{Lmnb}^{--}$ESCs to generate $\mathrm{Lmnb}^{-/} ; \mathrm{Lmnb2}^{-/} ; \mathrm{Lmna} \mathrm{t}^{\text {tml/t}}$ ESCs. To generate $\mathrm{Lmnb}^{-/} ; \mathrm{Lmnb2}^{-1-} ; \mathrm{Lmna}^{\text {tml/tm2 }}$ (referred to as lamin triple knockout (TKO) here) ESCs, the deletion was introduced again into $\mathrm{Lmnb1}^{-/} ; \mathrm{Lmnb}^{-/} ; \mathrm{Lmna}^{\mathrm{tmI/+}}$ ESCs. We confirmed homologous recombination at both the $5^{\prime}$ and $3^{\prime}$ arms by PCR (Figure 1B and 1C). mRNA expression from exons 1-5 of Lmna was not detected beyond the noise level in lamin TKO ESCs as judged by the RNAseq reads and reverse transcriptase (RT) PCR (Supplementary information, Figure S1A and S1B). Transcripts from the undeleted exon 1 in the mutant Lmna allele must be unstable due to a lack of polyA. However, RNA-seq indicates a low level of transcripts spanning exon 6-12 in TKO ESCs (Supplementary information, Figure S1A). They are likely derived from un-polyAed read-through transcription from the puromycine or hygromycine selection cassette that replaces exons 2-5. 
A
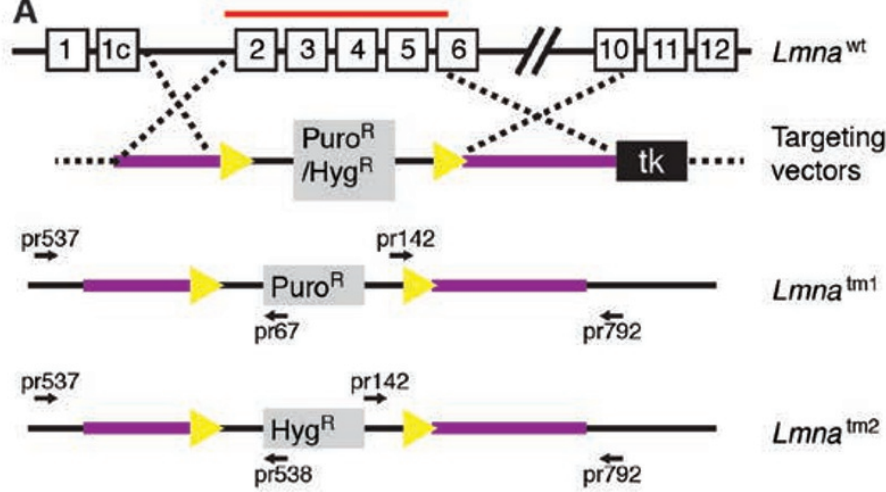

D

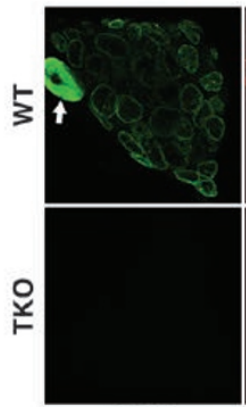

LA/C

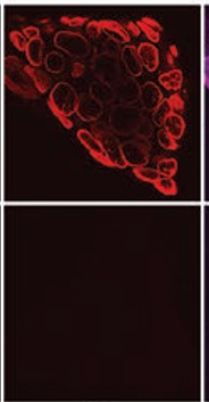

LB1

$\mathbf{F}$
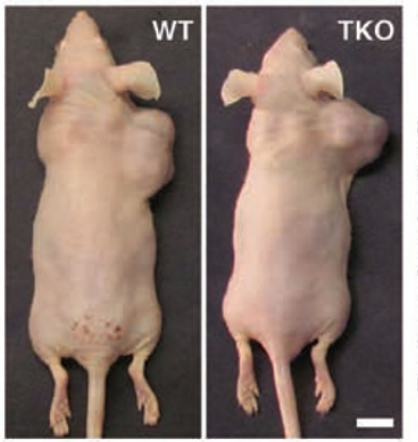

G
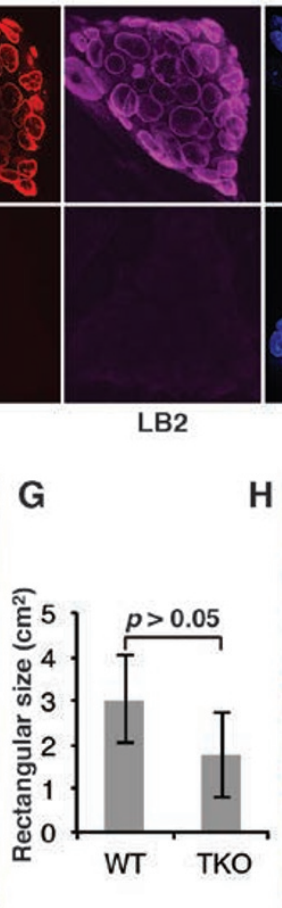

LB2

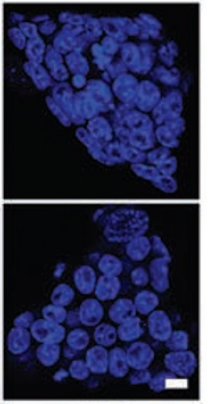

DNA

H

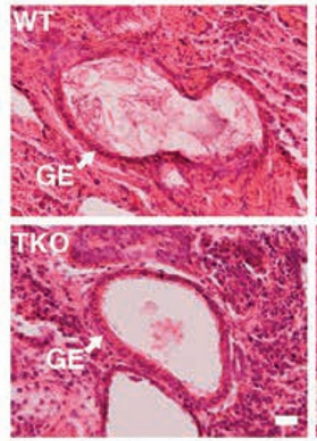

endoderm

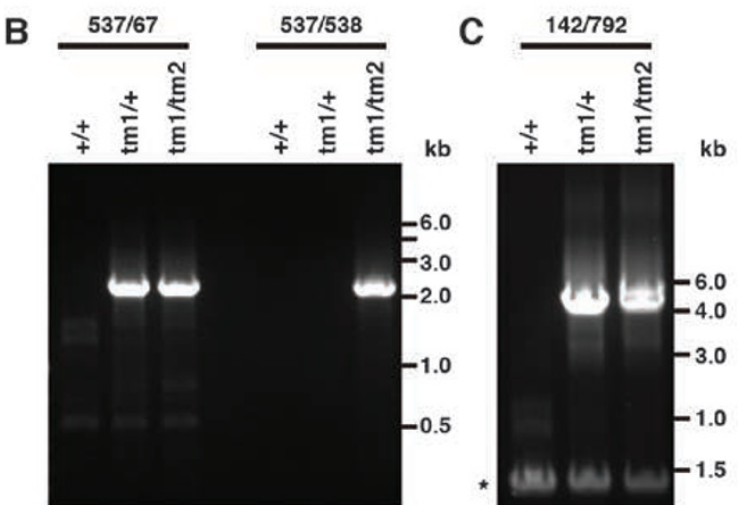

E
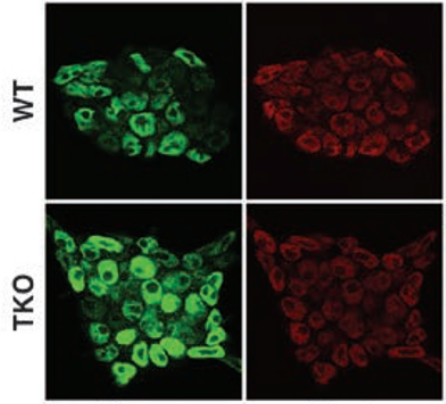

Oct4

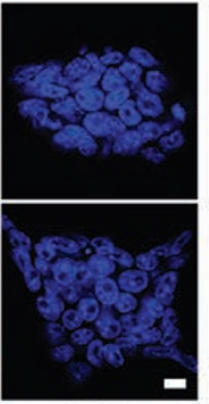

DNA

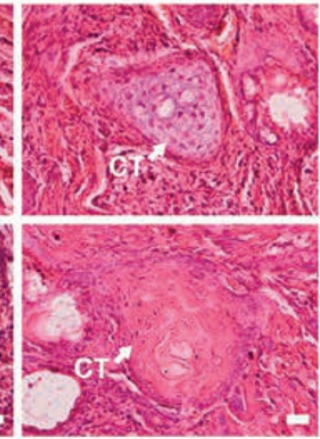

mesoderm

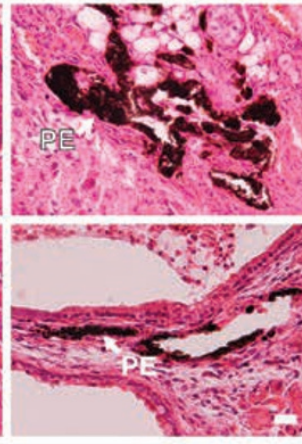

ectoderm

Figure 1 Characterization of lamin-null (TKO) ESCs. (A) Diagram of the targeting strategy. The $3.3 \mathrm{~kb}$ region (red line) from exon 2 through the first 14 bases of exon 6 in each $L m n a^{\text {wt }}$ allele was successively deleted using the two targeting vectors shown. To generate the $L m n a^{t m 1}$ or $L m n a^{t m 2}$ allele, a targeting vector containing either the puromycin or hygromycin resistant gene was introduced. Thick purple lines indicate the targeting arms corresponding to the genomic regions in the $L m n a$ gene. Yellow arrows represent the loxP sites. Puro ${ }^{R}$, puromycin resistance gene; Hyg $^{R}$, hygromycin resistance gene; TK, thymidine kinase gene; $\mathbf{t m}$, targeted mutation. The plots are not drawn to scale. (B and $\mathbf{C}$ ) Confirmation of homologous recombination of the $5^{\prime}(\mathbf{B})$ and $3^{\prime}(\mathbf{C})$ arms in two different Lmna mutant alleles. (B) Correct homologous recombination of the $5^{\prime}$ arms is indicated by the amplification of $2235 \mathrm{bp}$ and $2151 \mathrm{bp}$ PCR fragments from $L m n a^{t m 1}$ and $L m n a^{t m 2}$ alleles, respectively. The reverse primer of each pair targets allele-specific sequences. (C) Correct homologous recombination of the $3^{\prime}$ arms is indicated by the amplification of 4249 bp or 4695 bp PCR fragments from the $L m n a^{t m 1}$ or $L m n a^{t m 2}$ alleles, respectively. Primer pair for $3^{\prime}$ arms is common for both mutant alleles, generating two different fragment sizes in $L^{m n} a^{t m 1 / t m 2}$ allele. Asterisk marks nonspecific bands. Primer pairs used and genotypes for Lmna are indicated at the top of the panels $\mathbf{B}$ and $\mathbf{C}$. The relative positions of PCR primers in the Lmna gene are indicated in A. (D and E) Immunofluorescence staining of ESCs with antibodies against lamins (D) or pluripotency markers (E). LA/C, lamin-A/C; LB1, lamin-B1; LB2, lamin-B2. DNA was counterstained with Hoechst dye. Arrow in D indicates a MEF feeder cell exhibiting strong lamin-A/C expression. Scale bars, $10 \mu \mathrm{m}$. (F-H) Teratoma analyses. (F) Representative images of teratomas formed in the subcutaneous region of immune-deficient mice at 5 weeks post ESC injection. Scale bar, $1 \mathrm{~cm}$. (G) Quantification of the size of dissected teratomas. Error bars, SD; $n=5$. (H) H\&E staining of teratoma sections formed by wild-type (WT) and lamin-null (TKO) ESCs. Arrows point to the indicated tissues. GE, gut epithelium; CT, cartilage; PE, pigment epithelium. Scale bars, $20 \mu \mathrm{m}$. 
As the antibiotic cassettes contain a stop codon, readthrough transcripts are predicted to not express the C-terminus of lamin-A/C.

To examine whether lamin-A/C were expressed in lamin TKO ESCs, we used an antibody recognizing the C-terminal region of lamin-A/C. This highly sensitive antibody has been used to show the low level of expression of lamin-A/C in ESCs by Eckersley-Maslin et al. [6]. We found that lamin-A/C were missing from lamin TKOESCs but were expressed in wild-type ESCs (Figure 1D). This also confirms the lack of lamin- $\mathrm{A} / \mathrm{C}$ translation from the low level transcripts detected from exons 6-12 in lamin TKO ESCs. As expected, lamin-B1 and -B2 were expressed in wild-type ESCs, but not in lamin TKO ESCs (Figure 1D). As lamin TKO ESCs do not express any lamin, we conclude that these ESCs are lamin-null.

We further examined the lamin-null ESCs by immunostaining for the pluripotency markers, Nanog and Oct4. Both wild-type and lamin-null ESCs express similar levels of these proteins (Figure 1E). Immunofluorescence staining for nuclear pore complexes (NPC) and emerin showed grossly normal localization and distribution of these proteins in lamin-null ESCs compared to wild-type (Supplementary information, Figure S2A). Importantly, lamin-null ESCs exhibit growth rates (Supplementary information, Figure S2B) and euploidy (Supplementary information, Table S1) similar to those of wild-type ESCs. This demonstrates that the low level of lamin-A/ $\mathrm{C}$ expression in ESCs does not have a role in ESC selfrenewal. Furthermore, RNA-seq data indicates that genes known to regulate ESC pluripotency, proliferation, or lineage specification do not exhibit significant expression changes in lamin-null ESCs (data not shown). Therefore, lamins are not required for the basic proliferation and survival of ESCs.

An important feature of ESCs is the ability to differentiate into all embryonic cell lineages. We examined whether lamin-null ESCs can differentiate into fibroblasts, neural and cardiac lineages using established differentiation methods [7-9]. We found that both wildtype and lamin-null ESCs differentiated to fibroblasts as judged by cell morphology (Supplementary information, Figure S3A). As expected, a strong upregulation of lamin-A/C was observed during fibroblast differentiation of wild-type and lamin-B DKO ESCs. By contrast, no lamin-A/C was detected in lamin-null (TKO) cells even at 6 days of differentiation (Supplementary information, Figure S3B). Immunofluorescence staining of lamin$\mathrm{A} / \mathrm{C}$, -B1 and -B2 showed that these proteins were not expressed in lamin-null fibroblasts (Supplementary information, Figure S3C). We also found that laminnull ESCs could undergo differentiation into neural and cardiac lineages as judged by neural lineage marker expression (Supplementary information, Figure S4) and beating colonies hatched from embryoid bodies (data not shown), respectively. Therefore lamins are not required for in vitro differentiation of ESCs into fibroblasts, neural, or cardiac lineages.

Finally, we used a teratoma formation assay to analyze the differentiation potential of lamin-null ESCs. When injected subcutaneously into immune-deficient mice, both wild-type and lamin-null ESCs produced mature, cystic masses near the injected area (Figure 1F). We found that dissected teratomas formed by laminnull ESCs were similar to those formed by wild-type ESCs (Figure $1 \mathrm{~F}$ and $1 \mathrm{G}$ ). Histological analyses revealed that teratomas formed by both the wild-type and laminnull ESCs contained tissues representing the endoderm, mesoderm, and ectoderm (Figure 1H). Thus, lamin-null ESCs are able to differentiate into cells of all three germ layers.

We have shown previously that ESCs deleted of both lamin-B1 and -B2 genes undergo proper self-renewal and differentiation. Additionally, lamin-B DKO mice develop to birth, but die immediately after birth with severe defects in organs such as the diaphragm and the brain. As many cells in mice are known to express no or very low levels of A-type lamins during early embryonic development, we have proposed that lamins are not required for lineage differentiation or initial organogenesis. Instead, these proteins are required for the building of proper organ architecture. Although this idea is appealing because it narrows down the function of lamins for further dissection of their molecular mechanisms of action, the possibility exits that a small amount of A-type lamins in ESCs and potentially in all cell types could compensate for the loss of B-type lamins. Our findings reported here demonstrate that lamins are indeed not required for the self-renewal and differentiation of ESCs as judged by different in vitro differentiation models. This provides further support for a role of lamins in proper tissue building [10-12]. Our RNA-seq data indicates that $\sim 600$ genes do exhibit expression change upon lamin deletion. It would be interesting to further dissect the mechanism by which lamin may directly or indirectly influence these gene expressions in ESCs and whether such differential gene expression influences proper tissue building during development. It will also be important to further study whether animals without any lamin can develop to birth and whether different lamins are required for the building of different organs. The lamin-null (TKO) ESCs reported here should provide a great tool to study the function of different lamins in the context of tissue building.

Detailed methods are described in the Supplementary 
information, Data S1.

\section{Acknowledgments}

We thank O Martin for technical support, A Pinder for RNAseq, and CM Fan and the members of the Zheng lab for critical comments. This work is supported by HHMI, Ellison Medical Foundation, and R01 GM56312 (YZ).

\section{Youngjo Kim ${ }^{1}$, Xiaobin Zheng ${ }^{1}$, Yixian Zheng ${ }^{1}$}

${ }^{I}$ Carnegie Institution for Science, 3520 San Martin Dr., Baltimore, MD 21218 USA

Correspondence: Yixian Zheng

Tel: +1-410-246-3032; Fax: +1-410-243-6311

E-mail: zheng@ciwemb.edu

\section{References}

1 Butin-Israeli V, Adam SA, Goldman AE, et al. Trends Genet 2012; 28:464-471.

2 Worman HJ, Fong LG, Muchir A, et al. J Clin Invest 2009; 119:18251836.

3 Stewart C, Burke B. Cell 1987; 51:383-392.

4 Rober RA, Weber K, Osborn M. Development 1989; 105:365-378.

5 Kim Y, Sharov AA, McDole K, et al. Science 2011; 334:1706-1710.

6 Eckersley-Maslin MA, Bergmann JH, Lazar Z, et al. Nucleus 2013; 4:53-60.

7 Smith AG. Methods Cell Sci 1991; 13:89-94.

8 Yang X, Guo XM, Wang CY, et al. Methods Enzymol 2006; 418:267283

9 Conti L, Pollard SM, Gorba T, et al. PLoS Biol 2005; 3:e283.

10 Zuela N, Bar DZ, Gruenbaum Y. EMBO Rep 2012; 13:1070-1078.

11 Kim Y, McDole K, Zheng Y. Nucleus 2012; 3:256-262.

12 Chen H, Chen X, Zheng Y. Cell Stem Cell 2013; 13:73-86.

(Supplementary information is linked to the online version of the paper on the Cell Research website.) 